\title{
Time to refocus the NHS on quality and dignity of patient care: RCPE response to Mid Staffordshire
}

\author{
${ }^{1} \mathrm{NG}$ Dewhurst, ${ }^{2} \mathrm{MC}$ Jones, ${ }^{3} \mathrm{JA}$ Wilson \\ ${ }^{1}$ President; ${ }^{2}$ Vice President (Education E Standards); ${ }^{3}$ Vice President (Professional Standards), Royal College of Physicians of Edinburgh
}

\begin{abstract}
Earlier this month the Francis Report was published.' As doctors, and also as individuals with relatives who may have required hospital admission at some stage, we cannot help but be shocked and deeply saddened by the failings and poor standards of care in Mid Staffordshire which contributed to the premature deaths of hundreds of patients. Running to I,794 pages, the inquiry report makes harrowing and depressing reading for all with an interest in standards of care and presents a litany of failings which occurred at all levels (systemic, management, medical and nursing). Let us be under no illusion that the problems encountered in Mid Staffordshire were a localised, or isolated, happening. The contributing circumstances have the potential for this to occur in any hospital under pressure, and leave no room for complacency.
\end{abstract}

As Robert Francis QC observes in his introduction, it has unfortunately been the experience of too many inquiries that 'following an initial courtesy of a welcome and an indication that its recommendations will be viewed favourably, progress in implementation becomes slow or non-existent'. He also eloquently addresses the subject of hindsight, noting that in the Bristol Inquiry the word 'hindsight' appeared 120 times in the evidence and that 'benefit of hindsight' appears 378 times in the transcripts of the oral hearings of this Inquiry. We emphatically agree that the lessons learned here are too important to ignore, must be acted upon and result in cultural change to avoid repetition. If we do not, we will have failed the patients whose best interests we claim to represent.

The problems facing us, and particularly all of us involved in the provision of acute medical care in hospitals, are several, have been a number of years in the making and are interconnected (Figure I). The rapid rotation of trainees through the specialties, while working shifts and plugging rota gaps, has severely eroded the concept of medical teams. The European Working Time Regulations (EWTR) along with a run-through training policy that has limited the number of available medical registrars is further compounded by the increasing unpopularity of the acute medical take, the steady loss to other specialties (general practice, radiology, anaesthetics) and other countries (Australia/New Zealand). Changes to immigration rules have led to the virtual cessation of the availability of medical trainees from other countries to work in the NHS. The indirect effect of an improvement in the gender balance has seen an unanticipated increase in maternity leave and less than full-time working. When this is added to the reduced presence of general practitioners out of hours, increasing numbers of medical admissions and the reduction in acute hospital beds with resultant year-round boarding, the causes of malaise within the body politic of hospital doctors cannot be a mystery. Understanding these vicious circles and the pathogenesis of our condition is much less of a challenge than is the formulation of an effective treatment package.

The Francis Report makes 290 recommendations as a basis for change. It would not be feasible or desirable to cover all of these within the scope of this editorial. Instead, we would highlight the general recommendations which we believe must be supported by all stakeholders (Figure 2).

\section{SENIOR CARE AND REVIEW}

Many of the detailed recommendations also offer potential for improving standards, including the recommendation that 'hospitals should review whether to reinstate the practice of identifying a senior clinician who is in charge of a patient's case'. However, we believe this should not be a matter for local hospitals to consider and discount; instead this should be enshrined in national standards. The benefits of early senior review in reducing mortality, and of consultant-delivered care are clear and the requirement to have a named senior clinical lead responsible for individual patients could greatly assist efforts to improve continuity of care in increasingly fragmented clinical environments. ${ }^{2}$ Clearly, this will require moving towards a consultant presence seven days per week, over an extended working day, and this will have to be taken into account by workforce planners. The recent report from the Academy of Medical Royal Colleges and Faculties provides related standards which require to be implemented in the NHS. ${ }^{3}$

Historically, the NHS has relied heavily on the goodwill and commitment of consultants to work well beyond their contracted hours. However, there is evidence that this 'goodwill model' is not sustainable. ${ }^{4}$ Doctors cannot be expected to absorb additional commitments ad infinitum while continuing to provide high-quality patient care. This reality and the changing mood within the workforce, in which young doctors are increasingly 


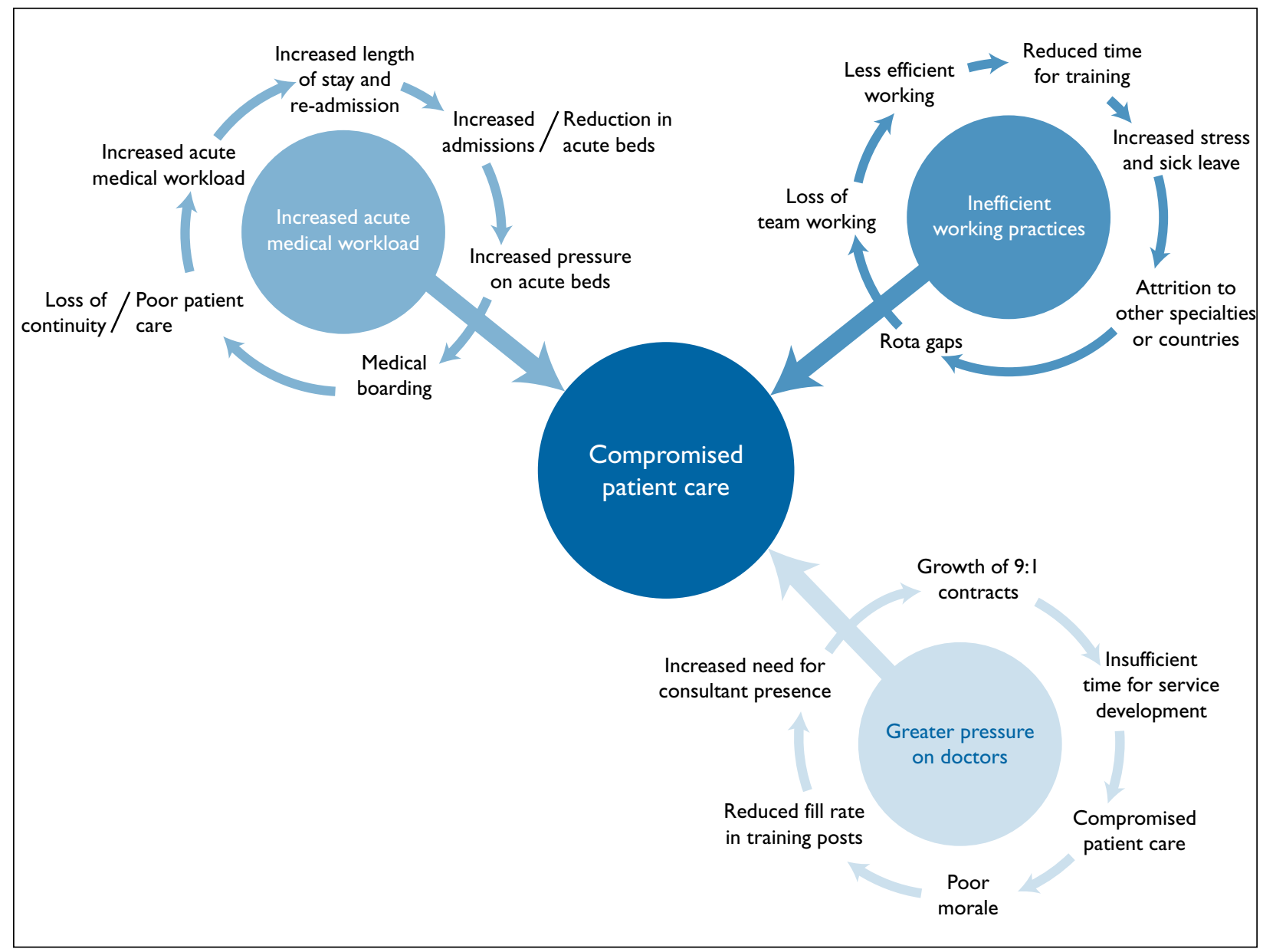

FIGURE I Vicious circles in the acute medical specialties

choosing alternative career paths away from the medical specialties, must also be taken into account when planning what level of workforce will be required to provide high-quality, safe patient care. In parallel, we must work to ensure that future generations of doctors are supported, do not become disengaged and less committed to delivering care and are prepared to work beyond hours as patients' needs require.

\section{STRENGTHENING TEAMWORKING AND ENGAGEMENT}

Coupled with increasing senior responsibility for patients is the need to establish more effective teamworking that recognises the particular challenges and pressures in nursing caused by high turnover in the workforce. It is also all too evident from the inquiry report and our experience of working within the NHS that many clinicians feel disengaged from management and are concerned that their clinical advice regarding best treatment for patients can be overruled by non-clinical managers. As such, we believe there is a pressing need to address the disconnect which has emerged and to improve clinical engagement with management.

\section{THE ROLE OF THE COLLEGES}

The report also recommends that the Colleges should be enlisted to provide the specialist expertise to support General Medical Council visits to inspect local education provision and the National Institute of Health and Clinical Excellence in developing procedures, metrics or guidance to assist compliance with the fundamental standards. We strongly support the continuing need for independent clinical input in monitoring performance in relation to service quality and outcomes.

We will continue to work with these statutory bodies, but we believe the Royal College of Physicians of Edinburgh (RCPE) has a much larger and wider role to play in improving standards of medical care. As Fellows and Members will be aware, the RCPE has become increasingly active in recent years in seeking to influence health policy for the benefit of patients. In Scotland, we do this directly through contact with the Scottish Government and are the only Scottish College engaging in an ongoing, cross-party basis with Members of the Scottish Parliament to inform the development of policy. Most recently, this has resulted in the announcement of a joint RCPE and Scottish Government initiative to reduce the level of boarding in Scottish hospitals 
- Foster a common culture shared by all in the service of putting the patient first;

- Develop a set of fundamental standards, easily understood and accepted by patients, the public and healthcare staff, the breach of which should not be tolerated;

- Provide professionally endorsed and evidencebased means of compliance with these fundamental standards which can be understood and adopted by the staff who have to provide the service;

- Ensure openness, transparency and candour throughout the system about matters of concern;

- Ensure that the relentless focus of the healthcare regulator is on policing compliance with these standards;

- Make all those who provide care for patients individuals and organisations - properly accountable for what they do and to ensure that the public is protected from those not fit to provide such a service;

- Provide for a proper degree of accountability for senior managers and leaders to place all with responsibility for protecting the interests of patients on a level playing field;

- Enhance the recruitment, education, training and support of all the key contributors to the provision of healthcare, but in particular those in nursing and leadership positions, to integrate the essential shared values of the common culture into everything they do;

- Develop and share ever improving means of measuring and understanding the performance of individual professionals, teams, units and provider organisations for the patients, the public, and all other stakeholders in the system.

FIGURE 2 Francis report: summary of general recommendations.'

following repeated advocacy of our concerns regarding the adverse impact this has on patient care and, in particular, on the frail elderly who are disproportionately affected by boarding and experienced some of the worst care in Mid Staffordshire. ${ }^{5}$

In Scotland we have also repeatedly voiced our concerns about the quality of official data used to inform medical workforce planning. These data have not reflected the contributions of the specialties to the acute medical take and an RCPE survey conducted last year found that $50 \%$ of respondents contributed more than $30 \%$ of their time to that activity. ${ }^{6}$ Without a practical understanding of how the acute medical specialties function, workforce planning projections will continue to be seriously flawed. Following presentation of these data, the Scottish Government has recognised this situation and the RCPE is now working with them, through our newly established Standards Unit, to ensure more informed workforce planning. In England we channel our policy-influencing activity through the Academy of Medical Royal Colleges and Faculties through which we continue to have a strong voice. We also believe that our activity in Scotland, in areas such as boarding, will have wider application throughout the whole of the UK.

\section{SERVICE PROVISION AND TRAINING}

In recent years there has been a steady erosion in the balance between service provision and training which has resulted in a reduction of the quality of training in the UK. This has the potential to compromise patient safety. Successive independent inquiries and reports have confirmed this problem, but have not delivered improvement. ${ }^{7,8}$ Against this background the RCPE published the Charter for Medical Training which we believe provides a firm basis for improving the quality of training. ${ }^{9}$ More recently the RCPE has responded to the ongoing Shape of Training Review which we believe will result in fundamental change in the UK. ${ }^{10}$ In our response to this we have highlighted the interconnected problems in the acute medical specialties which impact upon training and the quality of patient care and made a number of substantive recommendations including:

- that there is a rising need for all-age generalist expertise in hospital medicine to ensure patients have equitable access to services that are delivered efficiently and effectively in hospitals;

- the status and working patterns for general physicians and their trainees must be addressed urgently to combat an imminent crisis in the staffing of acute medical rota;

- patients welcome continuity of care and this could be delivered in some hospitals by the development of a cadre of consultant general physicians carrying lead responsibility for patients throughout their hospital stay and accessing other specialist input as required;

- acute medical specialties should require the great majority of trainees to dual accredit in general medicine, reflecting the continuing need for doctors in other medical specialties to contribute to acute unselected medical receiving; and

- the NHS requires fully trained clinical practitioners in general medicine to expand the availability of consultants seven days a week and for an extended working day.

As with the Francis Report, the Shape of Training Review has the potential to change medicine as we know it in the UK. We believe it imperative that training is not considered in isolation and that the vicious circles that impair our ability to deliver high-quality care in the acute medical specialties are broken.

\section{SO, HOW WILL THE RCPE RESPOND TO THE FRANCIS REPORT?}

Where there is evidence, we will continue to advocate the development of evidence-based, and more informed, health policy; where the evidence has not evolved sufficiently to permit such an approach, we will continue to build consensus based on emerging evidence and clinical experience. Only through doing this can we hope 
to influence standards of patient care. It is a sad irony that in the resource-constrained NHS a range of policy imperatives designed to make the NHS more patientcentred have regrettably measured activity and throughput rather than quality and contributed to a culture in which quality of care is not encouraged, supported or valued. This must change. Similarly if we are serious about driving up standards it is clear there is a need to give patients a louder, more systematised voice which would tell us what we need to know about our performance at the institutional level before it started to seriously fail. Related opportunities are available to link the work of College Lay Advisory Groups into the working of the NHS to further influence the development of a high standard of care. We also have a responsibility to encourage doctors to reflect repeatedly on their practice and to consider if we are being as compassionate and caring to patients as we can and are providing the standards of care which we would expect for ourselves and our families.

We will work with other stakeholders to ensure that there is greater transparency about standards of care, that doctors are encouraged and supported in raising legitimate concerns about poor standards of care and that these concerns will be shared with the appropriate bodies. The RCPE will be developing a confidential standards 'hotline' by which Fellows and Members who have witnessed poor quality care that is resistant to local change can raise concerns. Fellows and Members in the UK will also be encouraged to complete an RCPE survey regarding standards of care and reporting mechanisms to inform this workstream.

\section{REFERENCES}

I Final report of the Mid Staffordshire NHS Foundation Trust public inquiry [Internet]. London; 2013 [cited 2013 Feb 19]. Available from: http://www.midstaffspublicinquiry.com/report

2 Academy of Medical Royal Colleges. The benefits of consultant delivered care [Internet]. London: AOMRC; 2012 [cited $2013 \mathrm{Feb}$ 19]. Available from: http://www.aomrc.org.uk/about-us/news/item/ benefits-of-consultant-delivered-care.html

3 Academy of Medical Royal Colleges. Seven day consultant present care [Internet]. London: AOMRC; 2012 [cited 2013 Feb 19]. Available from: http://www.aomrc.org.uk/projects/seven-day-working.html

4 Federation of Royal Colleges of Physicians of the UK. Census of consultant physicians and medical registrars [Internet]. London: Federation of Royal Colleges of Physicians of the UK; 2010 [cited 2013 Feb 19]. Available from: http://www.rcplondon.ac.uk/ resources/2010-census-summary and $201 \mathrm{I}$ (Forthcoming).

5 Scottish Government and Royal College of Physicians of Edinburgh. Reducing ward moves: plans to reduce boarding in Scotland's hospitals [Internet]. Edinburgh RCPE; 2012 [cited 2013 Feb 19]. Available from: http://www.rcpe.ac.uk/press-releases/2012/reducing-wardmoves.php

6 Scottish Government. Reshaping the medical workforce in Scotland: consultation on specialty training numbers, 2013 and beyond, response from the Royal College of Physicians of Edinburgh, September 2012 [Internet]. RCPE: 2013 [cited 2013 Feb 19]. Available from: http:// www.rcpe.ac.uk/policy/20I/reshaping-the-medical-workforce-inscotland.php
We believe the RCPE is becoming increasingly influential through our approach to informing health policy. Central to this has been the support and contribution of our Fellows and Members who have given their time to support our data collection across a range of areas. Change of the magnitude required by the events in Mid Staffordshire will take time to deliver and be an uphill task. We need you and your colleagues' help in gathering information to support our activities, and if you have a view that you would like to be heard please email this to the_president@rcpe.ac.uk or attend one of our regional meetings to discuss in person.

We should be in no doubt that a response to this report is required from the Colleges and other medical organisations individually and collectively. Confidence in the quality of outcome achieved by the Health Service is a crucial part of the ability to be effective. We are fortunate to still be placed high in the public's esteem. Either we, as a profession, formulate and insist on the implementation of a credible plan, as a response to this latest evidence of a Health Service in crisis or we will have a less effective plan imposed upon us.

The need for strong medical leadership has never been greater. We believe the NHS has lost its focus, that now is the time for the NHS to re-commit to the central importance of high quality care and to foster a culture in which the delivery of this care is placed above all else. Nothing less than this is required to give patients the care and respect which they need to receive and we need to deliver.

7 Collins J. Foundation for excellence: an evaluation of the Foundation Programme [Internet]. London: Medical Education England; 2010 [cited $2013 \mathrm{Feb}$ 19]. Available from: http://www.mee.nhs.uk/ $\mathrm{pdf} / 401339$ MEE FoundationExcellence acc.pdf

8 Temple J.Time for training: a review of the impact of the European working time directive on the quality of training [Internet]. London: Medical Education England; 2010 [cited $2013 \mathrm{Feb}$ 19]. Available from: http:// www.mee.nhs.uk/PDF//4274\%20Bookmark\%20Web\%20Version.pdf

9 Royal College of Physicians of Edinburgh. Charter for medical training [Internet]. Edinburgh: RCPE; 20II [cited $2013 \mathrm{Feb}$ 19]. Available from: http://www.rcpe.ac.uk/policy/charter/rcpe-charterfor-medical-training.pdf

10 General Medical Council. Shape of training review: call for ideas and evidence: response from the Royal College of Physicians of Edinburgh, February 2013 [Internet]. Edinburgh: RCPE; 2013 [cited 2013 Feb 19]. Available from: http://www.rcpe.ac.uk/policy/2013/shape-oftraining-review.php 